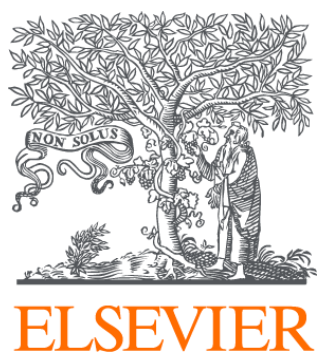

Since January 2020 Elsevier has created a COVID-19 resource centre with free information in English and Mandarin on the novel coronavirus COVID-

19. The COVID-19 resource centre is hosted on Elsevier Connect, the company's public news and information website.

Elsevier hereby grants permission to make all its COVID-19-related research that is available on the COVID-19 resource centre - including this research content - immediately available in PubMed Central and other publicly funded repositories, such as the WHO COVID database with rights for unrestricted research re-use and analyses in any form or by any means with acknowledgement of the original source. These permissions are granted for free by Elsevier for as long as the COVID-19 resource centre remains active. 


\section{Update on Emerging Infections: News From the Centers for Disease Control and Prevention}

Peter A. Cameron, MBBS, MD Timothy H. Rainer, MBBCh, MD

From the Department of Emergency Medicine, Chinese University of Hong Kong (Cameron, Rainer), and the Emergency Department, Prince of Wales Hospital (Cameron, Rainer), Hong Kong SAR, People's Republic of China.

\section{Section Editors}

David A. Talan, MD

Gregory J. Moran, MD

Olive View-UCLA Medical Center,

Sylmar, CA

Robert Pinner, MD

Centers for Disease Control and

Prevention, Atlanta, GA
Editor's note: This article is part of a regular series on emerging infections from the Centers for Disease Control and Prevention (CDC) and the EMERGEncy ID NET, an emergency department-based and CDCcollaborative surveillance network. Important infectious disease public health information with relevance to emergency physicians is reported. The goal of this series is to advance knowledge about communicable diseases in emergency medicine and foster cooperation between the front line of clinical medicine and public health agencies.

Update: Outbreak of Severe Acute Respiratory Syndrome-Worldwide, 2003

[Centers for Disease Control and Prevention. Update: Outbreak of severe acute respiratory syndrome-worldwide, 2003. MMWR Morb Mortal Wkly Rep. 2003;52:269-272.]

The Centers for Disease Control and Prevention (CDC) and the World Health Organization (WHO) are continuing to investigate the multicountry outbreak of unexplained atypical pneumonia referred to as severe acute respiratory syndrome (SARS). ${ }^{1}$ Pending development of confirmatory laboratory testing capacity, the CDC's interim suspected SARS case definition ${ }^{2}$ is based on clinic al criteria and epidemiologic linkage to other SARS cases or areas with community transmission of SARS. This case definition will be updated periodically as new information becomes available. Epidemiologic and laboratory investigations of SARS are ongoing. As of April 2, 2003, a total of 2,223 suspected and/or probable SARS cases have been reported to the WHO from 16 countries, including the United States. ${ }^{3,4}$ The reported SARS cases include 78 deaths (casefatality proportion: $3.5 \%$ ). This report summarizes SARS cases among US residents and surveillance and prevention activities in the United States.

DESCRIPTIVE EPIDEMIOLOGY As of April 2nd, the CDC had received 100 reports of suspected SARS cases from 28 states; 81 ( $81 \%)$ cases occurred among adults (Table). Of these 100 suspected cases, 94 (94\%) persons had traveled within the 10 days before illness onset to the areas listed in the case definition (revised on March 29th to include all of mainland China as an area with documented or suspected community transmission), 4 had household contact with a person with suspected SARS, and 2 were health care workers who provided medical care to a patient with suspected SARS. Manifestations of 
SARS have been relatively less severe among patients in the United States than among those reported elsewhere. A majority of US patients had normal chest radiograph results, and $23(23 \%)$ were reported to have pneumonia or respiratory distress syndrome on chest radiograph, thereby meeting the WHO case definition of a probable case. ${ }^{4}$ As of April 2 nd, of the $40(40 \%)$ patients who were hospitalized for more than 24 hours, $13(33 \%)$ remained hospitalized; one patient had required mechanical ventilatory support, and no deaths have been reported.

Reports on the clinical status of suspected SARS cases are being

Table.

Number and percentage of reported SARS cases, by selected characteristics-United States, 2003.

\begin{tabular}{ll}
\hline Characteristic & No. $(\%)(\mathrm{N}=100)$
\end{tabular}

\begin{tabular}{lc} 
Age, y & \\
$0-4$ & $9(9)$ \\
$5-17$ & $5(5)$ \\
$18-64$ & $71(71)$ \\
$\geq 65$ & $10(10)$ \\
Unknown & $5(5)$ \\
Sex & \\
Female & $48(48)$ \\
Male & $49(49)$ \\
Unknown & $3(3)$ \\
Race & \\
White & $50(50)$ \\
Black & $1(1)$ \\
Asian & $37(37)$ \\
Unknown & $12(12)$ \\
Exposure & \\
Travel & \\
Close contact & $94(94)$ \\
Health care worker & $4(4)$ \\
Hospitalized $>24$ h & $2(2)$ \\
Yes & \\
No & $40(40)$ \\
Unknown & $58(58)$ \\
Chest radiograph finding & $2(2)$ \\
Pneumonia or RDS & \\
Within normal limits & $23(23)$ \\
No or unknown results & $53(53)$ \\
Required mechanical ventilation & $24(24)$ \\
Yes & \\
No & $1(1)$ \\
Unknown & $93(93)$ \\
Uns & $6(6)$ \\
\hline &
\end{tabular}

RDS, Respiratory distress syndrome. *To mainland China, Hong Kong, Hanoi, or Singapore. received by state health departments and the CDC, and household and health care worker contacts are being monitored for the possibility of secondary transmission. Since SARS investigations in the United States began, some persons believed initially to have suspected SARS have been excluded on the basis of more complete clinical histories (eg, no documented fever or respiratory symptoms) or because of testing results that indic ated other etiologies. Alternative diagnoses have included infection with influenza virus, respiratory syncytial virus, Haemophilus influenzae, Streptococcus pneumoniae, and Staphylococcus aureus. Community transmission of SARS has not been identified in the United States; transmission to health care workers has been observed in one cluster involving 2 health care workers, compared with numerous reports of possible transmission to health care workers in other countries. ${ }^{5-7}$

\section{ENHANCED SURVEILLANCE FOR SARS RELATED TO TRAVEL}

As a precautionary measure, the WHO has recommended that persons traveling to Hong Kong and Guangdong Province of China consider postponing all but essential travel. The CDC has issued a travel advisory recommending that persons planning nonessential or elective travel to mainland China, Hong Kong, Hanoi, or Singapore consider postponing such travel until further notice. To detect possible SARS cases among travelers returning to the United States from these areas, the CDC and state and local health authorities have implemented enhanced surveillance. Since March 16th, notices (available in English, Chinese, Japanese, Korean, and Vietnamese) have been provided to approximately 220,000 passengers arriving in the United States on airline flights originating from China, Vietnam, and Singapore to inform disembarking passengers and crew about SARS. Persons disembarking from these countries are urged to monitor their health for 10 days after return, to seek medical care if they develop fever of more than $100.5^{\circ} \mathrm{F}$ $\left(>39.0^{\circ} \mathrm{C}\right)$ and cough or difficulty breathing within 10 days of travel, and to inform their health care providers about recent travel to regions where SARS cases have been reported.

\section{LABORATORY}

I N VESTIGATI ONS

Efforts are ongoing to characterize further the role of a previously unrecognized coronavirus in SARS. Polymerase chain reaction-based assays, isolation studies, electron microscopic studies, and histologic studies are being developed to detect virus in specimens from patients with suspected SARS. Indirect immunofluorescence antibody assays and enzyme immunoassays to anticoronavirus antibody as an indic ator of infection have been developed and are being applied to specimens from suspected SARS patients. Laboratory studies at the CDC and other laboratories in the WHO-organized SARS Laboratory Network have detected this new coronavirus in patients with SARS, which is consistent with an etiologic role in this disease. The CDC has detected human metapneumovirus from one patient with SARS, and other laboratories also have detected metapneumovirus from patients with SARS. ${ }^{6}$ The role of these viruses in the pathogenesis of SARS is unclear.

The number of SARS cases, as well as the number of countries reporting such cases, continues to increase worldwide. Transmission 
within hospitals and households continues in some areas, and transmission within communities (eg, Hong Kong) continues to be reported. In the absence of a complete understanding of how SARS is transmitted, efforts to limit transmission in the United States have focused on early identific ation of potential cases through surveillance and implementation of infection-control measures in health care settings and the community.

The CDC has developed interim infection-control guidelines for use in US health care and household settings. ${ }^{8}$ These recommendations are based on experience in the United States to date and will be revised as more information becomes available. Infection-control practitioners and clinicians providing medical care for patients with suspected SARS should consult the se guidelines frequently to keep current with recommendations.

Transmission in health care settings has been documented in several countries. Transmission to health care workers appears to have occurred primarily after close contact with symptomatic persons before recommended infection-control precautions for SARS were implemented. Because a primary strategy to reduce transmission in health care settings is early recognition and isolation of patients who might have SARS, triage practices in hospitals and ambulatory care settings might require reevaluation. The CDC guidelines for triage of potential SARS cases are available at http://www. cdc.gov/ncidod/sars/triage interim_guidance.htm.

In the United States, decisions to admit persons with suspected SARS to health care facilities should be based on clinical criteria. Patients with suspected SARS who are discharged should limit interactions outside the home and not go to work, school, out-of-home child care, or other public areas until 10 days after resolution of fever and respiratory symptoms. Additional guidance for these patients is available at http:// www.cdc.gov/ncidod/sars/icclosecontacts.htm.

The majority of US residents with SARS have recovered or stabilized clinically without specific antiviral therapy. The US case-fatality proportion is lower than that reported in some other countries. ${ }^{3}$ Possible explanations for this include differing case definitions among countries or differences in the sensitivity of surveillance, leading to identification in the United States of patients with less severe or early manifestations of infection or of a larger proportion of patients with other respiratory illnesses. Until confirmatory laboratory testing is available, the case definition will include clinical criteria more likely to identify potentially infectious persons. Various therapies, including antiviral agents (eg, oseltamivir or ribavirin) and corticosteroids, have been administered to patients with SARS, but the efficacy of these therapies has not been determined.

Health care providers of patients whose illness is consistent with the case definition for SARS should continue diagnostic evaluation for other causes of respiratory illness and, when appropriate, empiric therapy that includes activity against organisms associated with communityacquired pneumonia of uncertain etiology, including both typical and atypical respiratory pathogens. ${ }^{9}$ Health care providers who report suspected SARS cases should notify their state health departments if these patients receive confirmatory testing that indicates a diagnosis other than SARS. Information on suggested diagnostic testing and evaluation for persons with possible SARS is available athttp://www.cdc.gov/ ncidod/sars/diagnosis.htm.

The potential for transmission of SARS during airline travel is unknown. Transmission of other infectious agents (eg, Mycobacterium tuberculosis) during air travel has been demonstrated. ${ }^{10}$ When an airline flight crew reports a passenger with respiratory illness, quarantine officials might board the aircraft on arrival in the United States to assess whether the passenger's symptoms match the case definition of SARS and give the passenger information about follow up. If a passenger with suspected SARS is identified after passengers have disembarked, public health authorities will work with the airline to contact passengers and crew for information about the development of an illness suggestive of SARS. Although ill travelers have spread SARS rapidly across international borders, the proportion, if any, of persons who acquired SARS during international travel as a result of in-flight transmission is unknown.

Despite vigorous efforts to identify and isolate suspected cases, reducing transmission of the etiologic agents of SARS might be difficult. Understanding the epidemiology of respiratory pathogens such as those that cause SARS is challenging; approximately $40 \%$ to $60 \%$ of persons with pneumonia do not have a defined etiology, even when extensive testing for known respiratory pathogens is attempted. ${ }^{9}$ Minimizing transmission will require sustained attention to infection-control interventions within health care settings and the community. The development of laboratory testing techniques to identify infected persons rapidly will be an important step toward understanding and reducing transmission of SARS.

doi:10.1067/mem.2003.318 